\title{
Prevalence of Intestinal Parasite Infections among Children in the Day Care Centers of Gonbad-e Kavus County, North-Eastern Iran
}

\author{
Fatemeh Mesgarian, ${ }^{1}$ Aioub Sofizadeh,,${ }^{2,}$ Hamid Reza Shoraka, ${ }^{3}$ Hamid Reza Rahimi, ${ }^{1}$ Ali Hesari, ${ }^{1}$ \\ Najmeddin Soheili, ${ }^{1}$ and Farhad Badiei ${ }^{1}$ \\ ${ }^{1}$ Gonbad-e Kavus Health Center, Golestan University of Medical Sciences, Gorgan, IR Iran \\ ${ }^{2}$ Infectious Diseases Research Center, Golestan University of Medical Sciences, Gorgan, IR Iran \\ ${ }^{3}$ Maneh and Samalghan Health Center, North Khorasan University of Medical Sciences, Bojnurd, IR Iran \\ "Corresponding author: Aioub Sofizadeh, Infectious Diseases Research Center, Golestan University of Medical Sciences, Gorgan, IR Iran. E-mail: ayyubsofizadeh@gmail.com
}

Received 2017 May 13; Revised 2017 June 24; Accepted 2017 September 23.

\begin{abstract}
Background: Intestinal parasite infection is one of the major health problems in the world especially in the developing countries. Objectives: This study was an attempt to examine the prevalence of intestinal parasite infection in the day care centers in Gonbad-e Kavus.

Methods: In this cross sectional study, 932 children were recruited from 45 day care centers in Gonbad-e Kavus in Iran, through a survey and their stool samples were examined using direct wet mount, formalin-ether concentration and Trichrome staining techniques. Also, scotch tape slides were microscopically analyzed. Data were analyzed using percentage, frequency and Chi-square test using SPSS ver.16. Odds Ratio was used show the effect size of socio-demographic variables on the rate of intestinal parasite infection. Results: The prevalence of intestinal parasite infections among the children was $26.6 \%$. This study found a significant relationship between the children age, parent education and the place of residency with the rate of intestinal parasite infection $(P<0.05)$. The children in rural areas were 1.55 times more likely than the children in urban areas to be infected with intestinal parasites $(\mathrm{OR}=$ 1.55). Moreover, the results of this study showed that an increase in parent's education for one level decreased the risk of infection in their children by $6 \%(\mathrm{OR}=0.94)$. Also, with a rise in the age of participants for a year, the risk of infection increased by $44 \%(\mathrm{OR}=$ $1.44)$.

Conclusions: Due to the high rate of intestinal parasite infections among children, parents and child care workers need to learn about the various means by which parasites can be transmitted in day care centers.
\end{abstract}

Keywords: Intestinal Parasites, Protozoa, Day Care Centers, Iran

\section{Background}

Parasitic diseases as one of the most important health problems in the society, has a higher prevalence in developing countries and economically depressed communities [1, 2]. According to a report by the world health organization (WHO), about three billion of world population is infected with intestinal parasite [3, 4] and 450 million people, including children experience the adverse effects of intestinal parasitic disease [5]. A careful perusal of literature indicates that the prevalence rate of intestinal parasite infection is $7 \%-20 \%$ in developed countries while $30 \%-60 \%$ children in rural areas are affected by parasite infection in these countries [6-11]. Malnutrition, diarrhea, weight loss, anemia pose a mortal threat to human populations and cause about 16 million deaths in the developing countries every year $[1,12-14]$.
Previous surveys in Iran indicate that, the rate of intestinal parasitic disease in this country is between $4.7 \%$ $19.7 \%$ [15-19]. Compared with any other age group, children are more susceptible to intestinal parasite. The rate of infection among elementary school students is $22.2 \%-47.7 \%$ [20-22]. Children in day care centers are more prone to experience parasitic diseases. This could be associated with poor hygiene or presence of asymptomatic children [23, 24].

Parasitic diseases can be transmitted directly and indirectly. The direct transmission mostly occurs in crowded places such as kindergartens, dormitories, barracks and sanatoriums. In this way, the cysts of parasites are usually transmitted by hands or through contaminated tools or objects. The cysts can be also transmitted through water or by eating utensils $[1,25,26]$. Many children spend long 
hours together and use common things like toys. Handand object-to-mouth contacts with contaminated toys and objects can be a major culprit for the transmission of infection.

Different studies have been carried out on the prevalence of intestinal parasites in the day care centers, in Iran [22-24]. Some studies have documented a significant relationship between parent's education and the rate of intestinal parasite infection among their children [23, 24]. Low frequency of hand washing is reported to increase the risk of infection about 1.94 times [23]. So far, no study has been conducted on the prevalence of intestinal parasite infections in the day care centers in Gonbad-e Kavus. Therefore this study is an attempt to address this gap. The results of this study can help district health authorities and those who are in the charge of day care centers to make some measurements to prevent and control the spread of the parasites.

\section{Methods}

\subsection{Research Setting}

Gonbad-e Kavus $\left(55^{\circ} 18^{\prime}\right.$ E, $37^{\circ} 17^{\prime} \mathrm{N}$ ) lies up the north of Golestan province, Iran. In 2015, it had a population of 377,327 of which 38,860 were children under the age of 5 . (Statistics unit of Gonbad-e Kavus Health Center, unpublished data).

\subsection{Methodology}

This study is a cross-sectional study was carried out on the children of different day care centers in Gonbad-e Kavus. All children in 45 day care centers ( 23 urban and 22 rural centers) were studied through survey. From1191 children, 932 children between 1-9 years old were recruited to participate in this study.

Data on children's socio demographical information including parent's occupation, parent's education and the place of residence were collected through a questionnaire. To collect the morning stool samples of the participants, special containers with a particular number on, were given to parents and they were asked to send the samples to the laboratory of the health center in less than two hours. To diagnose Entrobius vermiculari,s scotch tape test was used. Three sets of samples were prepared, and then the samples were examined by direct wet mount, formalinether concentration and Trichrome staining techniques according to WHO protocol [27]. Also, the prepared scotch tape samples were observed under the microscope for the eggs of worms and trophozoites or cysts of protozoa. The obtained data was analyzed using SPSS version 16 software Descriptive statistics including the percentage and frequency were used to describe the survey data. Then association between socio demographic variables and intestinal parasite infection were analyzed using chi-square test. Variables with a P value less than 0.1 were included in the model of Multinomial Logistic Regression. The odds ratio at 95\% Confidence Interval was determined using Logistic Regression to evaluate the effect size of each variable on the rate of intestinal parasite infections.

\section{Results}

In this study, from 932 investigated children in different day care centers in Gonbad-e Kavus County, 2796 stool samples and scotch tape were collected and analyzed. Data showed that 248 (26.6\%) of the children were infected with the parasites. There was a significant relationship between some demographic characteristics of participants (children's age, parent's education and their place of residence) and the rate of infection to the parasites $(\mathrm{P}<0.05)$ However, there was no significant relationship between the children's gender and the risk for intestinal parasite infections $(P=0.239)$ (Table 1$)$.

Age, sex, mother's and father's educational level, place of residency, history of drug consumption and history of infection were studied using univariate regression model. Among the mentioned variables, mother's and father's education, place of residency and age were significantly associated with children infection rate. Analyzing the relationship between variables in multivariate regression model showed that the significance of mother's or father's educational level depends on the order of entering the data into this model. For example, when the data on father's level of education was entered first, mother's level of education did not show any significant relationship. To show the coeffect of these variables, their interactions were considered .An increase in parent education for one level decreased the risk of infection in their children by $6 \%(\mathrm{OR}=0.94)$. The children in rural areas were 1.55 times more likely than the children in urban areas to be infected with intestinal parasites with a rise in the age of participants for a year, the risk of infection increased by $44 \%(\mathrm{OR}=1.44)$ (Table 2$)$. One hundred fifty (16.1\%) children were infected with Enterobius vermicularis and 129 (13.8\%) were at least infected with a species of Protozoa. Thirty one (3.3\%) children were infected with both worm and protozoa (Table 3 ).

\section{Discussion}

The prevalence of intestinal parasitic infection among 932 children in different day care centers in Gobad-e Kavus, Iran was analyzed. Data analysis showed that $26.6 \%$ were 
Table 1. The Frequency of Intestinal Parasite Infection Among Children in the Day Care Centers: in Gonbad-e Kavus by Gender, Age, Parent's Education and Residency Status ${ }^{\mathrm{a}}$

\begin{tabular}{|c|c|c|c|c|c|}
\hline Variables & Subgroup & Infected Students & Healthy Students & Total & PValue \\
\hline \multirow{2}{*}{ Genus } & Male & $111(24.8)$ & $336(75.2)$ & $447(100)$ & \multirow{2}{*}{0.239} \\
\hline & Female & $137(28.2)$ & $348(71.8)$ & $485(100)$ & \\
\hline \multirow{3}{*}{ Age } & $<2$ & $5(11.9)$ & $37(88.1)$ & $42(100)$ & \multirow{3}{*}{0.001} \\
\hline & $3-4$ & 39 (18.9) & $167(81.1)$ & $206(100)$ & \\
\hline & $5-6$ & $204(29.8)$ & $480(70.2)$ & $684(100)$ & \\
\hline \multirow{4}{*}{ Mother's education } & Illiterate & $12(41.4)$ & $17(58.6)$ & $29(100)$ & \multirow{4}{*}{$<0.0001$} \\
\hline & Under-diploma & $101(38.4)$ & $162(61.6)$ & $263(100)$ & \\
\hline & Diploma & $73(22.2)$ & $256(77.8)$ & $329(100)$ & \\
\hline & Academic & $62(19.9)$ & $249(80.1)$ & $311(100)$ & \\
\hline \multirow{4}{*}{ Father's education } & Illiterate & $16(32)$ & $34(68)$ & $50(100)$ & \multirow{4}{*}{$<0.0001$} \\
\hline & Under-diploma & $105(40.9)$ & $152(59.1)$ & $257(100)$ & \\
\hline & Diploma & $67(22.5)$ & $230(77.5)$ & $297(100)$ & \\
\hline & Academic & $60(18.3)$ & $268(81.7)$ & $328(100)$ & \\
\hline \multirow{2}{*}{ Residence areas } & Urban & $150(22)$ & $530(78)$ & $680(100)$ & \multirow{2}{*}{$<0.0001$} \\
\hline & Rural & $98(38.9)$ & $154(61.1)$ & $252(100)$ & \\
\hline
\end{tabular}

${ }^{\mathrm{a}}$ Values are expressed as No. (\%).

Table 2. Multinomial Logistic Regression Analysis of the Factors Associated with the Risk for Parasite Infections Among the Children Different Day Care Centers in Gonbad-e Kavus

\begin{tabular}{lccc}
\hline Outcome & $\begin{array}{c}\text { Unadjusted Odds } \\
\text { Ratio }\end{array}$ & $\mathbf{P}>\mathbf{z}$ & $\begin{array}{c}\text { Adjusted Odds } \\
\text { Ratio }\end{array}$ \\
\hline Residence areas & 0.445 & $<0.0001$ & 1.55 \\
Age & 1.8 & $<0.0001$ & 1.444 \\
$\begin{array}{l}\text { Parent's } \\
\text { Education }\end{array}$ & 0.912 & $<0.0001$ & 0.94 \\
Constant & & & \\
\hline
\end{tabular}

infected with intestinal parasites. In the previous studies carried out in Iran, the reported rates were $68.1 \%, 47 \%, 36.6 \%$, $22 \%$ and $10 \%[22,24,28-30]$. The results of this study on the rate of infection with the intestinal parasites in Gobade Kavus were consistent with those reported in Shahroud city [22]. However, this rate was lower than the rate reported in Damghan and Bam [28, 29] but significantly higher than that in Illam and Yazd $[24,30]$. Such differences in the prevalence of different parasitic diseases could be associated with environmental, or socio -economic factors such as, the rate of humidity, temperature and dust, lack of health facilities in families or in day care centers; unhealthy handling of sewages; the residence place(rural or urban) and also the time of the study [22, 29, 31].

In the tested samples in this study; Enterobius vermic- ularis was observed in $16 \%$ of samples. In a study conducted in Bam [29], the rate was 15.9\% but in Semnan and Damghan [28], the rates were 8.15 and 33.8\% respectively. In the current study, no soil transmitted worms such as Ascaris, hook worms and strongyloides were observed in the tested samples. However, in the studies carried out in Venezuela and Keshmir [14, 32] Ascaris was reported to be the most prevalent worm. The rates of prevalence in these two countries were $28.4 \%$ and $49.3 \%$. Some studies in Illam and Bam $[24,29]$, showed the rate of infection with Ascaris lumbricus were $3.8 \%$ and $1.8 \%$ and this rate for strongyloides stercoralis was $0.8 \%$. This confirms that the children in our area of study are much healthier and are at the lower risk of infection.

In this study, 8 species of protozoa were observed in the tested samples. Blastocystis hominis had the highest frequency rate $7.9 \%$. This specie along with Giardia lamblia (2.8\%) and Dientamoeba fragilis (1.8\%) were among pathogenic protozoa observed in the study. Among nonpathogenic protozoa, including Endolimax nana had the highest infection rate (2.4\%), but Entamoeba coli, Iodamoeba buetschlii, Chilomastix mesnili and Trichomonas hominis had a lower frequency. In a study carried out in our country [33], the infection rates with G. lamblia were $14.7 \%$ and $15 \%$ children under the age of 10 . In a study conducted in Yazd [30], the infection with B. homonis and G. lamblia showed the highest rate, the infection rate with G. lamblia was $45.1 \%$ 
Table 3. The Frequency of Infection with Various Intestinal Parasites Among the Children Different Day Care Centers in Gonbad-e Kavus

\begin{tabular}{|c|c|c|c|c|c|c|c|c|}
\hline \multirow[t]{2}{*}{ Demographic Characteristic Species of Parasites } & \multicolumn{2}{|c|}{ Genus } & \multicolumn{2}{|c|}{ Residence Areas } & \multicolumn{3}{|c|}{ Age } & \multirow[t]{2}{*}{ Total Infected Children ${ }^{\mathrm{a}}$} \\
\hline & Male & Female & Rural & Urban & $2 \geq$ & $3-4$ & $5-6$ & \\
\hline Enterobius vermicularis & 85 & 65 & 64 & 86 & 2 & 22 & 126 & $150(1 / 16)$ \\
\hline Blastocystis hominis & 45 & 27 & 24 & 48 & 3 & 10 & 59 & $74(9 / 7)$ \\
\hline Giardia lamblia & 11 & 13 & 12 & 12 & 2 & 1 & 21 & $24(6 / 2)$ \\
\hline Dientamoeba fragilis & 11 & 4 & 11 & 4 & 0 & 1 & 14 & $17(8 / 1)$ \\
\hline Entamoeba coli & 3 & 5 & 5 & 3 & 0 & 0 & 8 & $8(8 / 0)$ \\
\hline Iodamoeba buetschlii & 1 & 0 & 0 & 1 & 0 & 0 & 1 & $2(2 / 0)$ \\
\hline Chilomastix mesnili & 0 & 2 & 0 & 2 & 0 & 0 & 2 & $1(1 / 0)$ \\
\hline Trichomonas hominis & 0 & 1 & 0 & 1 & 0 & 1 & 0 & $1(1 / 0)$ \\
\hline Total & 169 & 129 & 129 & 169 & 7 & 41 & 250 & $298(32)$ \\
\hline
\end{tabular}

${ }^{\mathrm{a}}$ Values are expressed as No. (\%).

in San Franciso and Venezuela [34]. However, the rate was quite lower in children (2.8\%) in our study. Consistent with the studies conducted in Iran [22, 28-30], the rates of intestinal parasite infection for boys and girls were $28.2 \%$ and $24.8 \%$, respectively. Data showed that the difference between boys and girls was not statistically significant. This means that gender doesn't have a big effect on intestinal parasite infection .This could be attributed to similar condition of boys and girls in our country.

In this study, a significant relationship was found between the age of children and their rate of infection with the intestinal parasites. However, in the other studies conducted in this country, there was no significant relationship between these two variables $[28,30]$. With an increase in the age of the participant, the risk of infection increased by $44 \%(\mathrm{OR}=1.44)$.In children smaller than two, the species of Enterobius vermicularis, Blastocystis hominis and Giardia lamblia were the major culprit for the infections, while in children over two, other parasites caused infection. This is probably is associated with easy transmission of parasite diseases through direct contact. In this study, a significant difference between the rate of and literacy level of parents was observed. These results were consistent with other studies carried out in Iran [24, 28-30]. An increase in parents' education for one level decreased the risk of infection in their children by $6 \%(\mathrm{OR}=0.94)$. Simply put, level of education can significantly contribute to prevention of children's infection to parasites. Perhaps, the knowledge of parents about the ways parasites can be transmitted or their awareness of the prevention techniques can decrease the risk for intestinal parasite infection among children. There was a significant relationship between the rate of and the children's residence place in this study. This result was echoed in studies conducted in Yazd, Bam, Illam and Damghan [24, 28-30]. Such a difference could be attributed higher health standard and knowledge of people in the urban areas. Also the children from urban areas were only infected with the parasites Iodamoeba buetchlii, Chilomastix mesnili and Trichomonas hominis.

\subsection{Conclusions}

Comparing the results of this study with the available research in literature shows that the general health condition in day care centers in Gonbad-e Kavus is satisfactory. However, the risk of intestinal parasite infection among children is still high. The prevention and health associated risk for parasitic infections require immediate attention of parents, district health authorities and those who work in day care centers. Due to the high rate of intestinal parasite infections among children, parents and child care workers need to learn about the various means by which parasites can be transmitted in day care centers.

\section{Acknowledgments}

Authors gratefully acknowledge that this study presents the results of research projects funded by the deputy of Research and Technology in Golestan University of Medical Sciences, Gorgan, Iran. (Project number: 35/265461, Executer: Fatemeh Mesgarian).

\section{Footnotes}

Authors' Contribution: All authors participated in the study and read the manuscript. Fatemeh Mesgarian 
and Aioub Sofizadeh designed study, developed the original idea and the protocol and preparated manuscript. Hamidreza Shoraka: data analysis;Farhad Badiei reviewed the literature. Hamidreza Rahimi, Ali Hesari and Najmeddin Soheili cooperated in child sampling and testing.

Conflicts of Interests: The authors have no conflicts of interest to declare.

Funding/Support: Golestan University of Medical Scinces.

\section{References}

1. Hotez PJ, Brindley PJ, Bethony JM, King CH, Pearce EJ, Jacobson J. Helminth infections: the great neglected tropical diseases. J Clin Invest. 2008;118(4):1311-21. doi:10.1172/JCI34261. [PubMed: 18382743].

2. Alva A, Cangalaya C, Quiliano M, Krebs C, Gilman RH, Sheen P, et al. Mathematical algorithm for the automatic recognition of intestinal parasites. PLoS One. 2017;12(4):e0175646. doi: 10.1371/journal.pone.0175646. [PubMed: 28410387].

3. Canete R, Diaz MM, Avalos Garcia R, Laud Martinez PM, Manuel Ponce F. Intestinal parasites in children from a day care centre in Matanzas City, Cuba. PLoS One. 2012;7(12):e51394. doi: 10.1371/journal.pone.0051394. [PubMed: 23236493].

4. Chirdan OO, Akosu JT, Adah SO. Intestinal parasites in children attending day care centers in Jos, Central Nigeria. Niger J Med. 2010;19(2):219-22. [PubMed: 20642093].

5. Mezeid N, Shaldoum F, Al-Hindi AI, Mohamed FS, Darwish ZE. Prevalence of intestinal parasites among the population of the Gaza Strip, Palestine. Ann Parasitol. 2014;60(4):281-9. [PubMed: 25706427].

6. Forsell J, Granlund M, Samuelsson L, Koskiniemi S, Edebro H, Evengard B. High occurrence of Blastocystis sp. subtypes 1-3 and Giardia intestinalis assemblage B among patients in Zanzibar, Tanzania. Parasites Vectors. 2016;9(1) doi:10.1186/s13071-016-1637-8.

7. Gonzalez-Moreno O, Domingo L, Teixidor J, Gracenea M. Prevalence and associated factors of intestinal parasitisation: a cross-sectional study among outpatients with gastrointestinal symptoms in Catalonia, Spain. Parasitol Res. 2011;108(1):87-93. doi: 10.1007/s00436-0102044-2. [PubMed: 20862495].

8. Rene BA, Stensvold CR, Badsberg JH, Nielsen HV. Subtype analysis of Blastocystis isolates from Blastocystis cyst excreting patients. Am J Trop Med Hyg. 2009;80(4):588-92. [PubMed: 19346381].

9. Roberts T, Barratt J, Harkness J, Ellis J, Stark D. Comparison of microscopy, culture, and conventional polymerase chain reaction for detection of blastocystis sp. in clinical stool samples. Am J Trop Med Hyg. 2011;84(2):308-12. doi: 10.4269/ajtmh.2011.10-0447. [PubMed: 21292905].

10. Li LH, Zhang XP, Lv S, Zhang L, Yoshikawa H, Wu Z, et al. Crosssectional surveys and subtype classification of human Blastocystis isolates from four epidemiological settings in China. Parasitol Res. 2007;102(1):83-90. doi: 10.1007/s00436-007-0727-0. [PubMed: 17912552].

11. El Safadi D, Gaayeb L, Meloni D, Cian A, Poirier P, Wawrzyniak I, et al. Children of Senegal River Basin show the highest prevalence of Blastocystis sp. ever observed worldwide. BMC Infect Dis. 2014;14:164. doi: 10.1186/1471-2334-14-164. [PubMed: 24666632].

12. Anderson TJC, Zizza CA, Leche GM, Scott ME, Solomons NW. The distribution of intestinal helminth infections in a rural village in Guatemala. Memorias do Instituto Oswaldo Cruz. 1993;88(1):53-65. doi: 10.1590/s0074-02761993000100010.

13. Cimino RO, Jeun R, Juarez M, Cajal PS, Vargas P, Echazu A, et al. Identification of human intestinal parasites affecting an asymptomatic peri-urban Argentinian population using multi-parallel quantitative real-time polymerase chain reaction. Parasit Vectors. 2015;8:380. doi: 10.1186/s13071-015-0994-z. [PubMed: 26183074].

14. Incani RN, Ferrer E, Hoek D, Ramak R, Roelfsema J, Mughini-Gras $\mathrm{L}$, et al. Diagnosis of intestinal parasites in a rural community of Venezuela: Advantages and disadvantages of using microscopy or RTPCR. Acta Trop. 2017;167:64-70. doi: 10.1016/j.actatropica.2016.12.014. [PubMed: 28007484].

15. Sarkari B, Hosseini G, Motazedian MH, Fararouei M, Moshfe A. Prevalence and risk factors of intestinal protozoan infections: a population-based study in rural areas of Boyer-Ahmad district, Southwestern Iran. BMC Infect Dis. 2016;16(1):703. doi: 10.1186/s12879-0162047-4. [PubMed: 27884121].

16. Nasiri V, Esmailnia K, Karim G, Nasir M, Akhavan O. Intestinal parasitic infections among inhabitants of Karaj City, Tehran province, Iran in 2006-2008. Korean J Parasitol. 2009;47(3):2658. doi:10.3347/kjp.2009.47.3.265. [PubMed:19724700].

17. Badparva E, Kheirandish F, Ebrahimzade F. Prevalence of intestinal parasites in Lorestan Province, West of Iran. Asian Pac J Trop Dis. 2014;4:S728-32.

18. Arani AS, Alaghehbandan R, Akhlaghi L, Shahi M, Lari AR. Prevalence of intestinal parasites in a population in south of Tehran, Iran. Rev Inst Med Trop Sao Paulo. 2008;50(3):145-9. [PubMed: 18604414].

19. Sayyari AA, Imanzadeh F, Bagheri Yazdi SA, Karami H, Yaghoobi M. Prevalence of intestinal parasitic infections in the Islamic Republic of Iran. East Mediterr Health J. 2005;11(3):377-83. [PubMed: 16602457].

20. Masoumeh R, Farideh T, Mitra S, Heshmatollah T. Intestinal parasitic infection among school children in Golestan province, Iran. PakJ Biol Sci. 2012;15(23):1119-25. [PubMed: 24261114].

21. Taheri F, Namakin K, Zarban A, Sharifzadeh G. Intestinal Parasitic Infection among School Children in South Khorasan Province, Iran.J Res Health Sci. 2011;11(1):45-50. [PubMed: 22911947].

22. Rahimi H, Dehghani M, Norouzi P, Fazli M. Frequncy of Giardia lamblia and Enterobius vermicularis infections in Shahroud Kindergartens, 2013. J Ardabil Univ Med Sci. 2015;15(1):7-14.

23. Atashnafas E, Ghorbani R, Peyvandi S, Imani S. Prevalence of oxyuriasis and some related factors in kindergarten and primary school children in urban areas of Semnan province (2005). Koomesh. 2007;9(1):67-74

24. Abdi J, Farhadi M, Aghaee S. Prevalence of Intestinal Parasites among Children Attending the Daycare Centers of Ilam, Western Iran. J Med Sci. 2014;14(3):143-6. doi:10.3923/jms.2014.143.146.

25. Sanchez A, Munoz M, Gomez N, Tabares J, Segura L, Salazar A, et al. Molecular Epidemiology of Giardia, Blastocystis and Cryptosporidium among Indigenous Children from the Colombian Amazon Basin. Front Microbiol. 2017;8:248. doi: 10.3389/fmicb.2017.00248. [PubMed: 28270802].

26. Ortiz C, Lopez MC, Rivas FA. [Helminth prevalence in a wastewater plant at El Rosal, Cundinamarca]. Rev Salud Publica (Bogota). 2012;14(2):296-304. [PubMed: 23250372].

27. World Health Organization . Manual of basic techniques for a health laboratory. Bull World Health Organ. 2004;82(3):384.

28. Heidari A, Rokni MB. Prevalence of intestinal parasites among children in day-care centers in Damghan-Iran. Iran $J$ Public Health. 2003;32(1):31-4.

29. Ahmad-Rajabi R, Varzandeh F, Arab M,Abbaszadeh A. Prevalence of intestinal parasite infections in the day care centers of bam.J Rafsanjan Univ Med Sci. 2003;2(2):102-11.

30. Anvari TMH, Mirjalili MM, Aghabagheri M. Prevalence of intestinal parasites in children attending day-care centers in yazd city, iran. $J$ Commun Health Res. 2014;3(2):96-102.

31. Rayan P, Verghese S, McDonnell PA. Geographical location and age affects the incidence of parasitic infestations in school children. Indian J Pathol Microbiol. 2010;53(3):498-502. doi: 10.4103/0377-4929.68292. [PubMed: 20699511]. 
32. Wani SA, Ahmad F, Zargar SA, Ahmad Z, Ahmad P, Tak H. Prevalence of intestinal parasites and associated risk factors among schoolchildren in Srinagar City, Kashmir, India. J Parasitol. 2007;93(6):1541-3. doi: 10.1645/GE-1255.1. [PubMed: 18314713].

33. Abasian L, Bazyar A, Shirbazo S, Sayehmiri K, Ahmad N. Prevalence of Giardia lamblia in Iran: a systematic review and Meta analysis study.
Res Med. 2013;36(5):111-6.

34. Cheng-Ng R, Castellano-Canizales JA, Diaz-Suarez O, VillalobosPerozo RE. Prevalence of giardiasis in day care centers in San Francisco municipality, state of Zulia, Venezuela. Investigacion clinica. 2002;43(4):231-7. 